\title{
The use of complementary alternative medicines in advanced cancer patients followed at home
}

\author{
Sebastiano Mercadante ${ }^{1,2}$ (D) Giuseppe Bellavia ${ }^{2} \cdot$ Alessio Lo Cascio $^{1} \cdot$ Marcella Dabbene $^{1} \cdot$ Grazia di Silvestre $^{3}$. \\ Alessandra Casuccio ${ }^{4}$ on behalf of the Regional home care program in Sicily SAMOT
}

Received: 1 June 2021 / Accepted: 15 September 2021 / Published online: 12 October 2021

(c) The Author(s), under exclusive licence to Springer-Verlag GmbH Germany, part of Springer Nature 2021

\begin{abstract}
Objectives To assess the use of complementary alternative medicines (CAM) in advanced cancer patients followed at home. Methods A consecutive sample of advanced cancer patients admitted to a home palliative care program was invited to participate in the study in a period of two months. Demographic characteristics, religious belief, education level, diagnosis, and socio-economic condition were recorded. Patients were asked about the use of CAM, considered to be a health-related treatment practiced outside the established health services. Information about CAM indications and those who prescribed or suggested CAM were also collected.

Results Two hundred and eighty-three advanced cancer patients followed at home were surveyed. Twelve patients $(4.2 \%)$ were receiving CAM. The indication and type of CAM were variable, as well as the costs. Given the low number of patients taking CAM, no further analysis was performed.

Conclusion A limited number of advanced cancer patients followed at home were using CAM. Further multicenter studies with a larger sample should be performed to provide information about such therapies, also including eventual benefits.
\end{abstract}

Keywords Advanced cancer $\cdot$ Palliative care $\cdot$ Home care $\cdot$ Complementary alternative medicine

The members of the Regional home care program in Sicily SAMOT are listed in Acknowledgment.

Key-message box 1 . What was already known?

Information about the use of CAM in advanced cancer patients

is poor. No information exists in patients followed at home.

2. What are the new findings?

The percentage of advanced cancer patients using CAM at home is less than 5\%, which is largely lower than that reported in other settings.

3. What is their significance?

In contrast with other findings evidenced in the general population of cancer patients, the use of CAM of patients admitted to a home care program in a Mediterranean region is negligible.

Sebastiano Mercadante terapiadeldolore@lamaddalenanet.it

Giuseppe Bellavia

g.bellavia@samotonlus.it

Alessio Lo Cascio

locascio.alessio@lamaddalenanet.it

Marcella Dabbene

dabbene.marcella@lamaddalenanet.it

Grazia di Silvestre

grazia.disilvestre@asppalermo.org

\section{Introduction}

In the last years, complementary and alternative medicine (CAM) has been widely used worldwide, varying from 10 to $76 \%$ [1-3]. Sixty-two per cent of Norwich population used CAM, including natural remedies (47.4\%), self-help practices $(29.1 \%)$, and therapies received from CAM providers (14.7\%), with a minority receiving CAM therapies from physicians (1.2\%). Women and younger people, lower education level and income were more likely to use CAM. Most of the participants found their use of CAM helpful
Alessandra Casuccio
alessandra.casuccio@unipa.it
1 Main Regional Center for Cancer Pain and Supportive/ Palliative Care, La Maddalena Cancer Center, Palermo, Italy
2 Regional Home Care Program, SAMOT, Palermo, Italy
3 Operative Palliative Care Unit, ASP 6, Palermo, Italy
4 Chair of Hygiene, University of Palermo, Palermo, Italy 
[4]. The prevalence of CAM use depends on what types of services, products, and self-help practices are included in the definition of CAM. There are specific conditions in which CAM is highly frequently used. Forty-five per cent of individuals diagnosed with insomnia in the USA have used a CAM therapy to treat their condition [5].

CAM is also a tempting option for cancer patients. Several experiences have been reported, particularly from Eastern countries. In patients with breast cancer, CAM was used by $70 \%$ of patients and was associated with higher education levels and household incomes, advanced cancer, and lower chemotherapy schedule compliance [6] and got information from television or radio [7]. More than $80 \%$ of all women with breast cancer reported using CAM, with $41 \%$ of them in a specific attempt to manage the disease [8]. Of interest, half of patients did not disclose their use of CAM to their oncologist, primarily, because the question was not raised in consultation. The most frequent CAM used by the participants were homeopathy (42\%), herbal medicine (27\%), acupuncture (22\%), vitamins (18\%), and massage therapy (15\%) [9].

A European survey examined CAM use and found that about $36 \%$ of cancer outpatients were using some form of CAM. A heterogeneous group of 58 therapies were identified as being used. Herbal medicines and remedies, homeopathy, vitamins/minerals, medicinal teas, spiritual therapies, and relaxation techniques were the most commonly CAM therapies. Younger people, females and patients with higher education level were more frequently using CAM. Friends, family, and media were the most frequent source of information which was less frequently provided by nurses and physicians. The reasons for using CAM were to increase their performance to fight cancer or to improve either physical and psychological well-being. Many patients were reported to achieve some benefit from CAM. Of interest, $38 \%$ of the Italian participants were using CAM [10].

Research in the area of CAM is scarce and with numerous therapies, we cannot be sure about efficacy or safety. The mechanisms of action are not well understood, and their use is often misled to medical professionals. Herbal medicines, for example, may have drug interactions such as cytochrome modulation, synergistic or additive effects [11]. In frail and elderly palliative care patients, the clinician should consider patients' comorbidities before recommending certain therapies, such as natural products $[12,13]$. A clinical practice guideline has been recently developed to standardize how oncologists address CAM use as well as to inform how individuals with cancer can be supported in making evidenceinformed decisions about CAM [14]. Current knowledge gaps urgently need to be filled. CAM is viewed as safe and holistic coupled with a view of conventional medicine as an aggressive and isolated treatment, despite the lack of evidence. There are multiple ideas woven together led patients toward CAM use. Such information is relevant in the process of care, even in the advanced stage of disease, as an understanding of patients' thought processes may aid health care professionals in initiating a dialogue about decision-making and potential side effects [15].

Information about the use of CAM in advanced cancer patients is lacking, particularly in Western European countries. A recent survey performed in France showed that a large number of advanced cancer patients $(90 \%)$ were using CAM in the previous six months or since their primary cancer diagnosis [16]. This is a very impressive data, considering the Health Care system, that is similar to that of Italy. The Healthcare system in Italy is based on a welfare model and approved treatments are free of charge or co-paid with a small fee. Clear information is not always obtainable and some patients may be confused by interested parties. CAM is fully paid for out of pocket, as it is offered outside the national Health Care System. There is a paucity of data examining CAM use in cancer patients receiving palliative care in Italy, resulting in a gap in scientific knowledge. In particular, data on advanced cancer patients followed at home are completely lacking. The aim of this study was to assess the use of CAM in advanced cancer patients followed at home.

\section{Methods}

\section{Study design}

This study employed an observational cross-sectional survey design.

\section{Setting}

The study was conducted in a regional home care program, SAMOT, which follows about 2000 patients per year in five provinces of Sicily, the largest region in Italy.

\section{Participants}

A consecutive sample of advanced cancer patients admitted to a home palliative care program was invited to participate in the study in a period of two months. Patients were approached by a member of the research team and invited to participate in the study. Only patients who agreed with the interview were surveyed.

\section{Data collection}

Demographic characteristics, religious belief, education level, diagnosis, and socio-economic condition (family income/month $<1000,1000<3000$ euros, $>3000$ euros) 
were recorded. Patients were asked if they were using CAM since home palliative care admission. CAM was considered a health-related treatment which was practiced outside the established health services and which is not practiced by authorized health personnel or the methods used are mainly used outside the national health care service [17]. Information about CAM indications and those who prescribed or suggested CAM were also collected.

\section{Statistics}

The sample size was based on the number of consecutive patients expected to be admitted to home palliative care with a diagnosis of cancer in two months and the numbers (n.52) of Italian patients included in the largest European study of CAM use in cancer patients, to provide a sample four times as many patients (at least 200 patients). Quantitative and qualitative data, including descriptive statistics, were analyzed for all items. Data were expressed as mean \pm standard deviation (SD), and categorical variables were presented as frequencies (percentages). Data were analyzed by IBM SPSS Software 22 version (IBM Corp., Armonk, NY, USA).

\section{Ethical clearance}

Approval to conduct the study was obtained from Azienda Sanitaria Locale (ASL n.6, Palermo). Participation was voluntary, and the participants were informed about the study both orally and in writing. All collected data were anonymized, handled, and stored in accordance with the tenets of the World Medical Association Declaration of Helsinki (2008).

\section{Results}

Two hundred and eighty-three advanced cancer patients followed at home were surveyed in the period taken into consideration. Twenty-one were not taken into consideration for evident cognitive disturbances. The mean age was 73 years (SD 11.6), and 149 (52.6\%) patients were males. Two hundred and seventy-seven patients were Italian citizens. One hundred and sixty-seven, 4, and 10 patients were catholic-anglican or evangelic, Jehovah's witnesses, and atheists, respectively.

Twelve patients $(4.2 \%)$ were receiving CAM. The principal patients' characteristics are listed in Table 1. The indication and type of CAM were variable, as well as the costs (from few euros to 500 euros per month). Given the low number of patients taking CAM, no further analysis was performed.

\section{Discussion}

Only a small percentage of advanced cancer patients followed at home were using CAM. People using CAM had different tumors, income, and education. Similarly, the indication, prescriber, or the type of CAM were largely variable, as well as the costs, which are not reimbursed by the Health Care System in Italy. The most frequent indication was about supportive care, rather than a curative intent.

Studies performed in palliative care patients seem to demonstrate a short-term benefit in symptom improvement from baseline with CAM, although a significant benefit was not found between the different groups of therapies [18]. Effectiveness results of complementary therapies in palliative care have been reported to be inconclusive, although patients may perceive them to be of some benefit [19].

The high prevalence of CAM use among cancer patients in Italy previously reported in a European survey [10] was attributed to a relatively high use of CAM in palliative care, in Northern Italy and in urban areas. In a regional study, the use of CAM in patients receiving chemotherapy was found to be modest, but still relevant (17\%) and prevalently observed among urbanites, women, and those with a higher level of education [20].

Few studies have assessed the use of CAM in patients receiving palliative care. In the USA, CAM was offered by over $40 \%$ of hospice care providers. About one-quarter of patients received CAM therapies during hospice care [21]. Despite increasing use in palliative care, many CAM therapies lack sufficient, high-quality evidence to support their use in the prevention and treatment of diverse conditions [22]. In a recent review evaluating the available evidence on the use of CAM in hospice and palliative care, CAM was found to provide a modest short-term benefit in patients with symptom burden [18].

In France, a country with many economic and cultural affinities with Italy, an observational cross-sectional survey conducted in inpatients and outpatients visiting the palliative care clinics, the majority of advanced cancer patients (90\%) had used CAM in the previous six months or since their primary cancer diagnosis [16]. The average out-of-pocket expenses associated with CAM use were about 157 euros/ month. The most common health professional visited was an aromatherapist (72.7\%), esoteric practitioner (38.6\%), osteopath $(28.6 \%)$, and naturopath (15.3\%). The most common CAM used were aromatherapy oils (33.7\%), homeopathy (30.0\%), and vitamins (29.4\%) [23].

Unfortunately, comparable data in advanced cancer patients followed at home are lacking. Thus, this is the first study assessing the use of CAM in a relatively large sample of advanced cancer patients followed at home. The comparison with patients using CAM after a cancer diagnosis or 


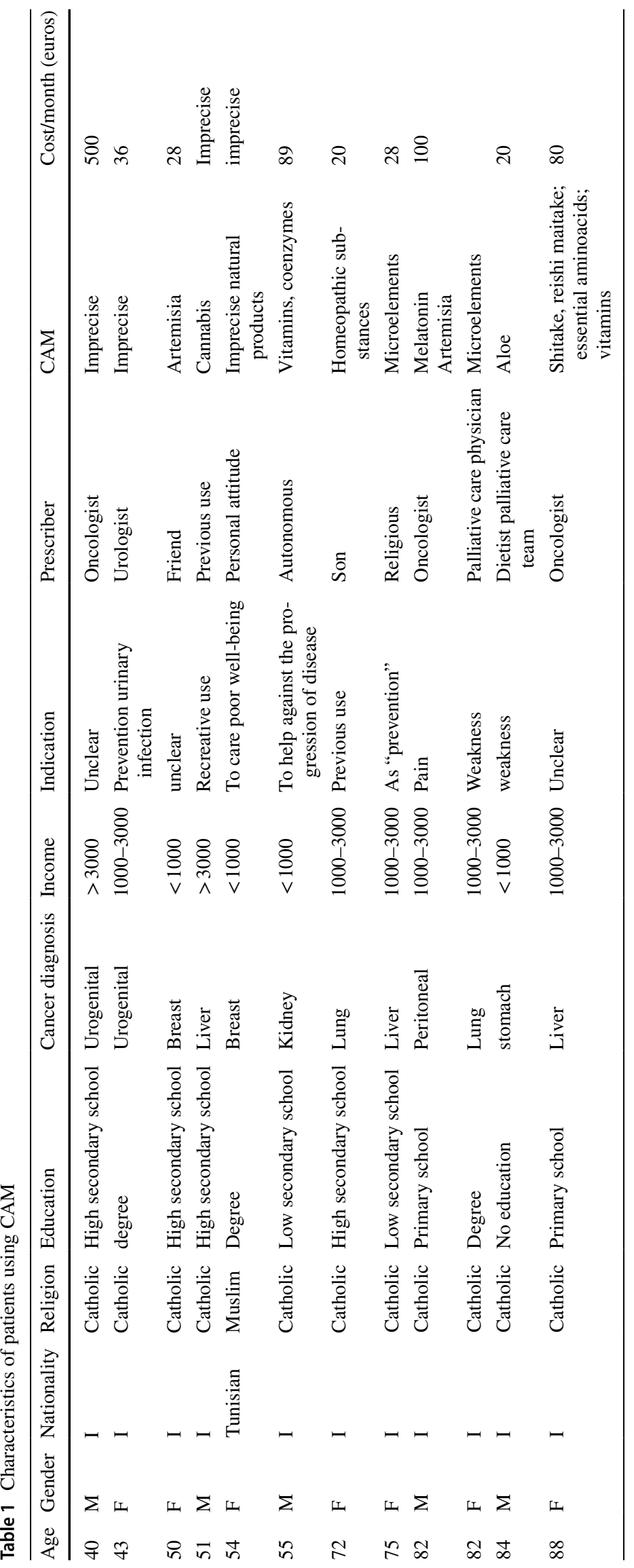


during the active treatment is not appropriate. Another possible reason could be that the study was performed during COVID-19 epidemics, possibly diverting attention toward the use of CAM.

This study has some limitations. While the number of patients was considered adequate in comparison with studies performed with palliative care patients or other settings, the low percentage of advanced cancer patients followed at home who were using CAM did not allow further analyses to assess possible variables associated with CAM use. Moreover, this is a single-center survey. There is the need of a large national study to draw definitive conclusion about the use of CAM in advanced cancer patients and possible associated variables. Finally, comparative studies with an adequate sample regarding the use of a specific treatment for a specific symptom would be of benefit for defining the role of CAM in palliative care.

\section{Conclusion}

A limited number of advanced cancer patients followed at home were using CAM. With such a low number of patients was not possible to investigate possible variables associated with this phenomenon. Further multicenter studies, more representative of Italian population with advanced cancer, should be performed to provide information about such therapies, also including eventual benefits.

Acknowledgements Acknowledgement for recruiting: Bruno Orlando MD (b.orlando@samotonlus.it), Tommaso Favara MD (t.favara@ samotonlus.it), Carla Scibilia MD (c.scibilia@samotonlus.it), Marina Gueci MD (m.gueci@samotonlus.it), Lucia Adamoli MD (1.adamoli@ samotonlus.it), Marco Mauceri MD (m.mauceri@samotonlus.it), Alessandro de Lisi MD (a.delisi@ samotonlus.it), Mario Lo Mauro MD (m.lomauro@samotonlus.it), Giuseppe Pirajno MD (g.pirajno@samotonlus.it).

Author contribution SM planning, conduct, responsible for the overall content; GB planning; AL data base; MD data base; GD administration; AC statistics.

\section{Declarations}

Data availability On request.

Code availability Excel - word.

Ethical approval Approval of the Institutional Review Board of ASP 6 Palermo.

Consent to participate Obtained consent to participate.

Consent for publication All patients provided their consent for publication.

Conflict of interest None.

\section{References}

1. Mbizo J, Okafor A, Sutton MA, Leyva B, Stone LM, Olaku O (2018) Complementary and alternative medicine use among persons with multiple chronic conditions: results from the 2012 National Health Interview Survey. BMC Complement Altern Med $18: 281$

2. Kemppainen LM, Kemppainen TT, Reippainen JA, Salmenniemi ST, Vuolanto PH (2018) Use of complementary and alternative medicine in Europe: health related and socio demographic determinants. Scand J Public Health 46:448-455

3. Peltzer K, Pengpid S (2018) Prevalence and determinants of traditional, Complementary and alternative medicine provider use among adults from 32 countries. Chin J Integr Med 24:584-590

4. Kristoffersen AE, Stub T, Salamonsen A, Musial F, Hamberg K (2014) Gender differences in prevalence and associations for use of CAM in a large population study. BMC Complement Altern Med 14:463

5. Ng YJ, Parakh ND (2021) Systematic review and quality assessment of complementary and alternative medicine recommendations in insomnia clinical practice guidelines. BMC Compl Med Ther 21:54

6. Chui PL, Abdullah KL, Wong LP, Taib NA (2014) Prayer-forhealth and complementary alternative medicine use among Malaysian breast cancer patients during chemotherapy. BMC Complement Altern Med 14:425

7. Knight A, Hwa YS, Hashim H (2014) Complementary alternative medicine use amongst breast cancer patients in the Northern Region of Peninsular Malaysia. Asian Pac J Cancer Prev 16:3125-3130

8. Boon HS, Olatunde F, Zick SM (2007) Trends in complementary/ alternative medicine use by breast cancer survivors: comparing survey data from 1998 and 2005. BMC Womens Health 7:4

9. Träger-Maury S et al (2007) Use of complementary medicine by cancer patients in a French oncology department. Bull Cancer 94:1017-1025

10. Molassiotis A et al (2005) Use of complementary and alternative medicine in cancer patients: a European survey. Ann Oncol 16:655-663

11. Jahromi B, Pirvulescu I, Candido KD, Knezevic NN (2021) Herbal medicine for pain management: efficacy and drug interactions. Pharmaceutics 13:251

12. Leong M, Smith TJ, Rowland-Seymour A (2015) Complementary and integrative medicine for older adults in palliative care. Clin Geriatr Med 31:177-191

13. Mansky PJ, Wallerstedt DB (2006) Complementary medicine in palliative care and cancer symptom management. Cancer $\mathbf{J}$ $12: 425-431$

14. Balneaves LG, Watling CZ, Hayward EN, Ross B, Taylor-Brown J, Porcino A (2021) Addressing complementary and alternative medicine use among individuals with cancer: an integrative review and clinical practice guideline. J Natl Cancer Inst 25:djab048

15. Sing H, Maskarinec G, Shumay DM (2005) Understanding the motivation for conventional and complementary/alternative medicine use among men with prostate cancer. Integr Cancer Ther 4:187-194

16. Steel A, Schloss J, Diezel H, Palmfren PJ, Maret JB, Filbet M (2020) Complementary medicine visits by palliative care patients: a cross-sectional survey. BMJ Support Palliat Care. https://doi. org/10.1136/bmjspcare-2020-002269

17. Lovdata. Act No. 64 of 27 June 2003 relating to the alternative treatment of disease, illness, etc. http://www.ub.uio.no/ujur/ulovd ata/lov-20030627-064-eng.pdf. Accessed 2 Mar 2021 
18. Zeng YS, Wang C, Ward KE, Hume AL (2018) Complementary and alternative medicine in hospice and palliative care: a systematic review. J Pain Symptom Manage 56:781-794

19. Armstrong M, Kupeli N, Flemming K, Stone P, Wilkinson S, Candy B (2020) Complementary therapy in palliative care: A synthesis of qualitative and quantitative systematic reviews. Palliat Med 34:1332-1339

20. Johannessen H, von Bornemann HJ, Pasquarelli E, Fiorentini G, Di Costanzo F, Miccinesi G (2008) Prevalence in the use of complementary medicine among cancer patients in Tuscany. Italy Tumori 94:406-410

21. Centers for Disease Control and Prevention (2007) Complementary and alternative therapies in hospice: the national home and hospice care survey: United States. https://www.cdc.gov/nchs/ data/nhsr/nhsr033.pdf. Accessed 2 May 2018
22. Ventola CL (2010) Current issues regarding complementary and alternative medicine (CAM) in the United States: Part 1: the widespread use of CAM and the need for better informed health care professionals to provide patient counseling. Pharm Ther 35:461-468

23. Filbet M, Schloss J, Maret JB, Diezel H, Palmgreen PJ, Steel A (2020) The use of complementary medicine in palliative care in France: an observational cross-sectional study. Support Care Cancer 28:4405-4412

Publisher's note Springer Nature remains neutral with regard to jurisdictional claims in published maps and institutional affiliations. 